\title{
The association between cognitive decline and incident depressive symptoms in a sample of older Puerto Rican adults with diabetes
}

\author{
Tyler Bell, ${ }^{1}$ Ana Luisa Dávila, ${ }^{2}$ Olivio Clay, ${ }^{1}$ Kyriakos S. Markides, ${ }^{3}$ Ross Andel ${ }^{4,5}$ \\ and Michael Crowe \\ ${ }^{1}$ Department of Psychology, University of Alabama at Birmingham, Birmingham, Alabama, USA \\ ${ }^{2}$ School of Public Health, University of Puerto Rico, San Fuan, Puerto Rico \\ ${ }^{3}$ Department of Preventive Medicine $\mathcal{E}$ Community Health, University of Texas Medical Branch, Galveston, Texas, USA \\ ${ }^{4}$ School of Aging Studies, University of South Florida, Tampa, Florida, USA \\ ${ }^{5}$ International Clinical Research Center, St. Anne's University Hospital, Brno, Czech Republic
}

ABSTRACT

Background: Older Puerto Rican adults have particularly high risk of diabetes compared to the general US population. Diabetes is associated with both higher depressive symptoms and cognitive decline, but less is known about the longitudinal relationship between cognitive decline and incident depressive symptoms in those with diabetes. This study investigated the association between cognitive decline and incident depressive symptoms in older Puerto Rican adults with diabetes over a four-year period.

Methods: Households across Puerto Rico were visited to identify a population-based sample of adults aged 60 years and over for the Puerto Rican Elderly: Health Conditions study (PREHCO); 680 participants with diabetes at baseline and no baseline cognitive impairment were included in analyses. Cognitive decline and depressive symptoms were measured using the Mini-Mental Cabán (MMC) and Geriatric Depression Scale (GDS), respectively. We examined predictors of incident depressive symptoms (GDS $\geq 5$ at follow-up but not baseline) and cognitive decline using regression modeling.

Results: In a covariate-adjusted logistic regression model, cognitive decline, female gender, and greater diabetes-related complications were each significantly associated with increased odds of incident depressive symptoms $(p<0.05)$. In a multiple regression model adjusted for covariates, incident depressive symptoms and older age were associated with greater cognitive decline, and higher education was related to less cognitive decline $(\mathrm{p}<0.05)$.

Conclusions: Incident depressive symptoms were more common for older Puerto Ricans with diabetes who also experienced cognitive decline. Efforts are needed to optimize diabetes management and monitor for depression and cognitive decline in this population.

Key words: diabetes, Hispanic, older adults, cognition, depressive symptoms

\section{Introduction}

Approximately $9.3 \%$ of the US population has diabetes, with older persons having higher rates than the general population (CDC, 2014). Aging with diabetes represents a major public health concern and underscores the need for better

\footnotetext{
Correspondence should be addressed to: Michael Crowe, Department of Psychology, University of Alabama at Birmingham, Holley Mears Bldg. 111, 1530 3rd Ave. S., Birmingham, Alabama 35294-2100, USA. Phone: +1-205 934-0231. Email: mgcrowe@uab.edu. Received 15 Sep 2016; revision requested 6 Nov 2016; revised version received 10 Apr 2017; accepted 13 Apr 2017. First published online 17 May 2017.
}

understanding of diabetes-related health outcomes in older adults. Specifically, there is evidence that people with diabetes are at higher risk for vascular problems such as myocardial infarction and stroke as well as cognitive decline due to vascular dementia and Alzheimer's disease (Xu et al., 2010; CDC, 2014). Because older adults are already at higher risk for these conditions, an aging population with diabetes may be even more vulnerable to cardiovascular and cognitive problems. The issue of an aging population is particularly relevant in Puerto Rico, where there was a $28 \%$ increase in adults aged 65 years and over between 2000 and 
2010, almost double the $15 \%$ increase seen in the USA during the same period (U.S. Census Bureau, 2011).

Furthermore, there is a recognized disparity in the prevalence of diabetes, with Hispanics at a higher risk of diabetes than non-Hispanic whites. For instance, Mexican American adults have an approximately $45 \%$ higher prevalence of diabetes compared to non-Hispanic whites (Kendzor et al., 2014). Recently, the Center for Disease Control (CDC, 2014) reported that Puerto Ricans (14.8\%) and Mexican Americans (13.9\%) have the highest rates of diabetes in Hispanic populations, considerably higher than the prevalence among nonHispanic whites in the USA (7.6\%). Additionally, certain minority populations including Hispanics have been found to have worse diabetes control compared to whites, not explained by differences in healthcare utilization, education or socioeconomic status (Harris et al., 1999). Worse diabetes control is related to long-term complications and makes efforts to improve treatment in this group paramount. One important factor that may contribute to poor diabetes control in this population is depression.

Studies have found that $12-20 \%$ of those with diabetes meet diagnostic criteria for major or subclinical depression, respectively (Ciechanowski, 2011), though comorbid depression may be even higher in Hispanic adults. For example, prevalence of depression was found to be $39 \%$ and $41 \%$ for Hispanic adults with diabetes in the USA and Mexico, respectively (Mier et al., 2008). It is important to note that the relationship between diabetes and depression may be bidirectional. Golden et al. (2008) found that the risk of developing diabetes increased for every pointincrease in depressive symptoms longitudinally, though this association became non-significant after controlling for sociodemographic, lifestyle, metabolic, and inflammatory factors. Conversely, there was an increased risk of incident depressive symptoms in those with diabetes, which remained significant in fully adjusted models. Thus, the presence of depressive symptoms in diabetes seems to increase with progression of the disease itself, and this relationship may be more pronounced in Hispanic persons who have both higher prevalence of depression and diabetes (CDC, 2014).

Depressive symptoms may have a negative impact on health outcomes in persons with diabetes since treatment is highly reliant on selfmanagement (Solanki et al., 2009). A person with diabetes must often make lifestyle changes that can be challenging and add to the burden of coping with the disease (Ciechanowski, 2011). Depression is a prime concern for diabetes-related health outcomes because it can negatively affect diabetes self-care (Egede and Osborn, 2010). It is recommended that individuals with diabetes adhere to a regimen that includes frequent blood glucose monitoring, medication (for some), regular physician visits, and proper dietary intake and exercise. Depression is associated with reduced self-efficacy, or perceived ability to successfully manage diabetes (Cherrington et al., 2010). In turn, lowered selfefficacy is associated with less self-care behavior, poorer blood glucose control, and poorer treatment adherence (Bernal et al., 2000; Sacco et al., 2005; Sarkar, Fisher, \& Schillinger, 2006; Gao et al., 2013). Black et al. (2003) found that in an elder Mexican American population, diabetes increased the risk of death, microvascular complications, and disability, but this risk of negative outcomes was even higher for those with more depressive symptoms (Black et al., 2003). Diabetes and depression together may have a synergistic effect on worsened health outcomes in older Hispanics with diabetes. Therefore, it is imperative to understand predictors of depressive symptoms within older Hispanic populations with high prevalence of diabetes.

Cognitive decline may be an important indicator of depression risk in diabetes. Studies have found greater levels of cognitive decline in older adults with diabetes (Fontbonne et al., 2001), and there is an accelerated conversion from Mild Cognitive Impairment (MCI) to dementia among those with diabetes (Xu et al., 2010). Over time, lower cognitive function may lead to worse diabetes control and increased caregiver burden (Munshi et al., 2006; Cukierman-Yaffe et al., 2009; Leroi et al., 2012). While it is often difficult to disentangle depression and cognitive impairment, depression has been linked to many of the same biological pathways as cognitive decline, including neural damage via hypothalamic pituitary adrenal dysregulation and chronic inflammation (Golden, 2007; Strachan et al., 2011). Richard et al. (2013) reported that depression was related to concurrent MCI but was not predictive of future incidence, possibly due to a shared causal manifestation.

Within a high-risk sample of Puerto Ricans (i.e. older adults with diabetes), we hypothesized that cognitive decline would be related with greater incidence of clinically significant depressive symptoms. Also, we examined whether age, education, gender, and health comorbidity had similar associations with both depressive symptoms and cognitive decline as outcomes in this sample of older Puerto Ricans with diabetes. 


\section{Methods}

\section{Participants}

The Puerto Rican Elderly: Health Condition (PREHCO) study is a longitudinal populationbased study of older adults in Puerto Rico. The study was conducted in two waves using a probability-based sampling technique to select homes with at least one adult over 60 years of age across the entire mainland of Puerto Rico. The first wave occurred from 2002 to 2003, with the second wave occurring from 2006 to 2007. The overall response rate was $93.9 \%$ and there were no significant differences between those who responded and those who did not respond (McEniry and Palloni, 2010). For the current study, participants were included in analyses if they reported a diagnosis of diabetes and completed cognitive testing and information on depressive symptoms at baseline and at 4-year follow-up.

Overall, 4,291 participants were recruited for PREHCO; 408 were not interviewable and 3,883 (90.5\%) completed cognitive testing at baseline. Of these, 75 had incomplete data and 292 participants had cognitive impairment (7.5\%). Individuals with suspected cognitive impairment, defined by scores less than 11 on the Mini-Mental Cabán $(M M C)$, were administered informant interviewee questionnaires in lieu of self-report. An informantreport parallel measure of depressive symptoms was not included, resulting in exclusion from the current study. There was a potential sample of 3,516 participants without cognitive impairment at baseline with information on depressive symptoms. There were 456 participants who died between baseline and follow-up, and 408 who were lost to follow-up, resulting in a sample of 2,652 without baseline cognitive impairment who completed follow-up cognitive testing. A total of 2,561 had complete data on variables of interest at followup, with $680(26.6 \%)$ participants who had diabetes at baseline. Our longitudinal analytic sample consisted of those with diabetes but without substantial baseline depressive symptoms $(n=$ 480 ), defined as having 5 or more depressive symptoms reported on the Geriatric Depression Scale (GDS; Friedman et al., 2005).

\section{Measures}

Demographics and health problems including diabetes were self-reported. Depressive symptoms were measured using a Spanish language version of the GDS, which was developed to improve detection of depression in older adults by relying less on somatic complaints and using a yes/no response format for symptoms (Yesavage et al.,
1983). The commonly used 15 -item scale (GDS15) has been shown to have acceptable reliability (Cronbach's $\alpha=0.77$; Friedman et al., 2005) and has convergent validity with other scales of depression, including the Hamilton Self-Rating Depression Scale and the Cornell Scale of Depression in Dementia (both $r$ 's $=0.77$; Kørner et al., 2006). The yes/no format of the GDS-15 was designed to decrease the cognitive demand of Likert scales. The scores range from 0 to 15 , with 15 indicating the highest number of depressive symptoms. For the current study, a cut-off of 5 was used to determine substantial depressive symptoms because a recent meta-analysis showed this cutoff to have the best sensitivity and specificity to predict clinically diagnosed depression across different languages including Spanish (Pocklington et al., 2016), and there was good sensitivity ( $80.7 \%)$ and acceptable specificity for this cut-off among Hispanic older adults (68.7\%; Aguilar-Navarro et al., 2007). Incident depressive symptoms were defined as scores surpassing the cut-off at followup but not at baseline.

The MMC was utilized at baseline and follow-up to assess global cognitive functioning. The MMC was designed to be more appropriate for use in Hispanic populations and those with low education levels compared to simple Spanish translation of the Mini-Mental State Examination (MMSE; Sánchez-Ayéndez et al., 2003). Scores on the MMC can range from 0 to 20 and items reflect orientation (day of week, date), verbal memory (immediate and delayed recall of three words), visual memory (immediate recall; draw complex figure after viewing for $15 \mathrm{sec}$ ), executive function (clock drawing, abstraction), and comprehension (follow three-step command).

The MMC was found to have superior sensitivity and specificity compared to the MMSE for detecting clinically diagnosed dementia in a clinicbased study of older adults in Puerto Rico (Sánchez-Ayéndez et al., 2003). To determine baseline cognitive impairment, MMC scores were regressed on years of age, female gender, years of education, and self-reported reading ability (yes/no). An expected score for each participant was calculated using the intercept and beta weights from the baseline regression model. Participants with MMC scores 1.5 standard deviations (SD) or more below the predicted score were classified as having cognitive impairment at baseline and not included in the longitudinal analytical sample of 480 participants. Cognitive decline was measured by subtracting follow-up MMC scores from baseline scores.

We included two health comorbidity measures in analyses: diabetes-related complications and 
Table 1. Characteristics of the sample with diabetes by presence of significant depressive symptoms

\begin{tabular}{|c|c|c|c|c|c|c|c|}
\hline & \multicolumn{3}{|c|}{ BASELINE GDS $<5(N=480)$} & \multicolumn{4}{|c|}{ BASELINE GDS $\geq 5(N=200)$} \\
\hline & RANGE & $M(\mathrm{SD})$ & $N(\%)$ & RANGE & $M(\mathrm{SD})$ & $N(\%)$ & $\mathrm{P}$ \\
\hline Female & & & $287(59.8)$ & & & $141(70.5)$ & 0.009 \\
\hline Male & & & $193(40.2)$ & & & $59(29.5)$ & \\
\hline Age & $60-89$ & $69.12(6.35)$ & & $60-92$ & $69.98(7.27)$ & & 0.139 \\
\hline Education (years) & $0-16$ & $8.46(4.52)$ & & $0-16$ & $7.39(4.63)$ & & 0.005 \\
\hline Vascular comorbidity & $0-4$ & $1.05(0.87)$ & & $0-4$ & $1.41(0.93)$ & & $<0.001$ \\
\hline Diabetes complications & $0-4$ & $0.92(0.92)$ & & $0-4$ & $1.51(1.03)$ & & $<0.001$ \\
\hline
\end{tabular}

Note: $t$-test and $\chi^{2}$ analyses are used to determine p-values for difference between those with and without baseline depressive symptoms.

vascular comorbidity. Diabetes-related complications were measured as a summed index score of self-reported history of problems with circulation, vision, foot sores, amputations, and kidney disease due to diabetes (range 0-4). Vascular comorbidity was measured as a summed index score of selfreported history of cardiovascular events including myocardial infarction, hypertension, congestive heart failure, and stroke (range 0-4).

\section{Statistical analysis}

First, we used $t$-tests and $\chi^{2}$ analyses to determine differences in the sample with diabetes by presence of significant depressive symptoms at baseline. Next, to examine predictors of incident depressive symptoms, we used a logistic regression model that included age, gender, education, vascular comorbidity, diabetes complications, cognitive decline, and baseline cognitive performance. We also examined predictors of cognitive decline using multiple linear regression. Cognitive decline was regressed on incident depressive symptoms, baseline cognitive function, age, gender, education, vascular comorbidity, and diabetes complications. Last, sensitivity analyses were conducted to discern whether a higher GDS-15 cut-off would lead to different findings. Analyses were conducted using SAS Version 17 software (SAS Institute, Inc., Cary, NC, USA, 2014).

\section{Results}

Health and demographic characteristics of the sample, separated by presence of substantial depressive symptoms at baseline, are shown in Table 1. Overall, those with substantial depressive symptoms at baseline were more likely to be female and had more diabetes-related complications as well as vascular comorbidities $(\mathrm{p}<0.05)$ compared to participants without depressive symptoms. Average years of education was lower for those with substantial depressive symptoms (7.4 years) compared to those with low depressive symptoms (8.5 years). In our longitudinal analytic sample of participants with no substantial depressive symptoms at baseline, average cognitive decline was 0.89 points $(\mathrm{SD}=2.33)$ and $25.22 \%$ showed a decline of at least three points on the MMC between baseline and follow-up.

Results from logistic regression modeling with incident depressive symptoms as the outcome are shown in Table 2. Cognitive decline was a significant predictor of increased depressive symptoms; for every additional point of decline in cognitive score, there was a $19 \%$ increased odds of incident depressive symptoms at followup (95\% CI: 1.07-1.33). Higher baseline cognitive performance was related to lower odds of incident depressive symptoms (OR $=0.80,95 \%$ CI: 0.70 $0.93)$. Women had $64 \%$ greater odds of incident depressive symptoms (95\% CI: 1.01-2.66) compared to men. There were no significant associations between incident depressive symptoms and age, education, or vascular comorbidities. However, diabetes-related complications were related to greater odds of incident depressive symptoms $(\mathrm{OR}=1.46 ; 95 \% \mathrm{CI}: 1.14-1.86)$. After $z$-score standardization, cognitive decline had a similar size of association as diabetes complications (OR $=$ 1.55 and 1.45 , respectively) with incident depressive symptoms.

Interactions were added to the model shown in Table 2 to determine if the relationship between cognitive decline and incident depressive symptoms was moderated by any of the covariates. Results indicated that the association between cognitive decline and incident depressive symptoms did not significantly vary by gender, age, education, baseline MMC, diabetes complications or vascular complications (all p's $>0.05$ ).

Next, cognitive decline from baseline to followup was regressed on incident depressive symptoms, baseline cognitive function, age, gender, education, diabetes-related complications, and vascular comorbidities (see Table 3). Results showed 
Table 2. Odds ratios for predictors of incident depressive symptoms in participants with diabetes

\begin{tabular}{|c|c|c|c|}
\hline \multirow[b]{2}{*}{ PREDICTOR(s) } & \multicolumn{2}{|c|}{$\begin{array}{c}\text { ODDS OF INCIDENT } \\
\text { DEPRESSIVE SYMPTOMS }\end{array}$} & \multirow[b]{2}{*}{$\mathrm{P}$} \\
\hline & OR & $95 \% \mathrm{CI}$ & \\
\hline Cognitive decline & 1.19 & $1.07-1.33$ & 0.001 \\
\hline Baseline MMC & 0.80 & $0.70-0.93$ & 0.002 \\
\hline Age & 0.97 & $0.94-1.01$ & 0.159 \\
\hline Female gender & 1.64 & $1.01-2.66$ & 0.045 \\
\hline Education & 1.01 & $0.96-1.07$ & 0.617 \\
\hline Diabetes-related complications & 1.46 & $1.14-1.86$ & 0.003 \\
\hline Vascular comorbidity & 1.01 & $0.77-1.32$ & 0.966 \\
\hline
\end{tabular}

Note: $\mathrm{OR}=$ odds ratio; $\mathrm{CI}=$ confidence interval.

Table 3. Predictors of cognitive decline in participants with diabetes

\begin{tabular}{|c|c|c|c|c|}
\hline & \multicolumn{4}{|c|}{$\operatorname{PREDICTOR}(s)$} \\
\hline & $B$ & $S E$ & $\beta$ & $P$ \\
\hline Intercept & -13.87 & 1.51 & 0.00 & $<0.001$ \\
\hline Incident depressive symptoms & 0.79 & 0.24 & 0.13 & 0.001 \\
\hline Baseline MMC & 0.74 & 0.05 & 0.58 & $<0.001$ \\
\hline Age & 0.05 & 0.02 & 0.12 & 0.002 \\
\hline Female gender & -0.30 & 0.20 & -0.06 & 0.131 \\
\hline Education & -0.15 & 0.02 & -0.27 & $<0.001$ \\
\hline Diabetes-related complications & -0.08 & 0.11 & -0.03 & 0.471 \\
\hline Vascular comorbidity & 0.13 & 0.12 & 0.04 & 0.263 \\
\hline
\end{tabular}

Note: $B=$ unstandardized beta coefficient; $\beta=$ standardized beta coefficient.

significant associations between cognitive decline and incident depressive symptoms, baseline cognitive status, age, and education level. Older age was associated with greater cognitive decline $(b=0.05$, $\mathrm{p}<0.01)$, and more years of education was related to less cognitive decline $(b=-0.15, \mathrm{p}<0.001)$. Incident depressive symptoms were associated with greater cognitive decline $(b=.79, \mathrm{p}<0.01)$.

To address the issue of whether using a different GDS-15 cut-off would change results, we conducted sensitivity analyses using more conservative cutoffs $(7,8$, and 9). In logistic regression models with incident depressive symptoms as the outcome, the relationship between cognitive decline and incident depressive symptoms remained statistically significant with a GDS-15 cut-off of $7(\mathrm{OR}=1.10$, 95\% CI: $1.01-1.21)$ and $8(\mathrm{OR}=1.14,95 \% \mathrm{CI}$ : 1.03-1.25). Using a GDS-15 cut-off of 9, the pvalue for cognitive decline was increased to 0.06 $(\mathrm{OR}=1.10,95 \% \mathrm{CI}: .99-1.23)$.

\section{Discussion}

Substantial depressive symptoms were common in this sample of older Puerto Rican adults with diabetes. At baseline, approximately 30\% of the sample with diabetes also had high depressive symptoms. An additional $26 \%$ of those without significant depressive symptoms at baseline reported elevated depressive symptoms (GDS $\geq 5$ ) 4 years later. Consistent with hypotheses, cognitive decline was significantly related to an increased risk of incident depressive symptoms after controlling for demographics and health problems, adding to research suggesting shared cognitive and mental health changes in persons aging with diabetes (Richard et al., 2013). Most of the research in this area has specifically focused on depressive symptoms as a predictor of cognitive function (e.g. Paterniti et al., 2002; Rosenberg et al., 2010). However, this study highlights the need to also consider how changes in cognition may relate to subsequent depressive symptoms in older adults with diabetes.

Baseline comparisons revealed that individuals with significant depressive symptoms reported more diabetes complications and vascular comorbidities. Depressive symptoms have been linked to increased risk of cardiovascular disease and diabetes complications in prior research (e.g. Lin 
et al., 2010). Notably, the group with high depressive symptoms at baseline had greater frequency of females and individuals with lower levels of education. This is consistent with other research in older adults, where women have been found to have a higher prevalence of depression assessed via self-reported depressive symptoms and clinical diagnoses (Steffens et al., 2000; Anderson et al., 2001). Lower education has also been associated with higher depressive symptoms in previous research (Zahodne et al., 2014). These differences may help identify older individuals with diabetes who are at greatest risk for developing depression.

This research contributes to our understanding of the interrelationship between diabetes, cognitive function, and depressive symptoms. While depressive symptoms may be a risk factor for cognitive decline, our findings showed that cognitive decline is also predictive of incident depressive symptoms in older Hispanic adults with diabetes. The pathophysiological mechanisms for these relationships are not completely understood and could not be examined in the current study; however, the role of inflammatory pathways has been proposed as central to diabetesdepression-cognition associations (e.g. Marioni et al., 2010). Diabetes-induced neural damage, potentially caused by both chronic inflammation and insulin resistance, may make older adults more vulnerable to cognitive and mental health problems (Strachan et al., 2011).

While there was an association between cognitive decline and incident depressive symptoms, there were different relationships between these outcomes and demographic and health variables. For example, female gender and diabetes-related complications were significantly associated with increased depressive symptoms but not cognitive decline. Older age and less education were associated with cognitive decline but not incident depressive symptoms. There is ample research in the general older adult population showing that individuals with higher education have lower risk of dementia (Meng and D'Arcy, 2012). One explanation for the relationship between education and cognitive decline involves the concept of cognitive reserve, which is the ability to cope with neural damage through the recruitment of compensatory neural networks or pre-existing cognitive strategies (Stern, 2013). Even in the context of aging with diabetes, people with greater education may take longer to show signs of cognitive decline.

Limitations of this study include the use of self-report measures of health and assessment at only two time points. Incident depressive symptoms were determined using a cut-off on a self-report measure of depression and not by clinical evaluation. However, the GDS is a widely used and well-validated measure of depressive symptoms in older adults (D'ath et al., 1994). The measures used in this study were designed to be quickly administered, but without compromising reliability and validity, allowing for increased feasibility of data collection in large epidemiologic samples. Because the current study included two time points, we could not examine potential non-linear trends over time or utilize more complex longitudinal modeling techniques. Also, it should be noted that associations between depressive symptoms and cognitive functioning may be underestimated due to exclusion of adults with cognitive impairment at baseline.

Current guidelines by the American Diabetes Association (ADA) recommend that physicians screen and monitor older adults with diabetes for cognitive impairment and depression (American Diabetes Association, 2016). Though not recommended for all older adults, screening is recommended for adults with diabetes who report cognitive difficulties, impairments in activities of daily living, or high depressive symptoms (Pottie et al., 2015; ADA, 2016). Screening can help clinicians detect possible cases of cognitive impairment and depression who should then be referred to specialists for further testing and possibly treatment. Screening and referral for issues such as depression and cognitive impairment presents unique challenges in Puerto Rico, where the healthcare system has been described as being on the brink of collapse (Roman, 2015). Medicare reimbursement rates in Puerto Rico are substantially less compared to states in the USA and many physicians and other healthcare professionals have left the island over the past decade. In addition, quality of care for Medicare Advantage enrollees in Puerto Rico was found to be substantially worse compared to quality for enrollees in the mainland USA (Rivera-Hernandez et al., 2016). Thus, hope for improvement in agerelated health outcomes in Puerto Rico will be largely dependent on quality and availability of healthcare on the island.

Last, what can healthcare professionals do to prevent cognitive decline or reduce depressive symptoms in older adults with diabetes? Optimizing glycemic control may prevent substantial cognitive decline before the age of 70 in those with diabetes (Messier, 2005), and cardiovascular care is also thought to be important for preventing cognitive decline in this population (Takeda et al., 2010). In addition, getting older adults to engage in moderate exercise may reduce risk of cognitive decline and depressive symptoms. Several exercise 
interventions have been shown to improve cognitive function in older adults (Bherer et al., 2013) and resistance training (45 minutes, three times per week, over 16 weeks) has been shown to reduce depressive symptoms in older Puerto Rican adults specifically (Lincoln et al., 2011). Though challenging, such interventions may be especially important in older Puerto Rican adults who show the lowest rates of physical activity compared to other Hispanic subpopulations and non-Hispanic whites (Hajat et al., 2000), and exercise interventions show feasibility across different levels of cognitive dysfunction and frailty (Bherer et al., 2013). Regarding psychological interventions to reduce depressive symptoms, cognitive behavioral therapy, interpersonal psychotherapy, problem-solving therapy, and behavioral activation are effective treatments for depression in Hispanics (Cabassa and Hansen, 2007; Collado et al., 2016), especially when tailored to specific Hispanic cultures (Interian and DíazMartínez, 2007). For example, Rosselló et al. (2008) found that modified cognitive behavioral therapy and interpersonal psychotherapy emphasizing cultural values (e.g. familismo) were effective at reducing depressive symptoms in a Puerto Rican sample. Further, the ADA (2016) recommends collaborative care models, and in Puerto Rico, a collaborative model where physicians and mental health professionals worked together to coordinate antidepressant treatment and counseling provided better reduction of depressive symptoms than usual care (Vera et al., 2010). Still, any strategies for improving diabetes management and treating depression for older Puerto Ricans will only be successful to the extent that further erosion of the healthcare system on the island can be avoided.

\section{Conflict of interest}

None.

\section{Description of authors' roles}

Tyler Bell conducted data analyses and wrote the manuscript. Ana Luisa Dávila performed data collection, helped to prepare the dataset, and revised the manuscript critically for important intellectual content. Olivio Clay, Kyriakos Markides, and Ross Andel assisted in conceptualization of the study and contributed to the manuscript by revising it critically for important intellectual content. Michael Crowe assisted in conceptualizing the study, conducting data analyses, and writing the manuscript.

\section{Acknowledgments}

This work was supported in part by National Institute on Aging (NIA) Grants R21 AG045722 and P30AG022838. The content is solely the responsibility of the authors and does not necessarily represent the official views of NIA or the National Institutes of Health.

\section{References}

Aguilar-Navarro, S. G., Fuentes-Cantú, A., Ávila-Funes, J. A. and García-Mayo, E. J. (2007). Validity and reliability of the screening questionnaire for geriatric depression used in the Mexican health and age study. Salud Pública de México, 49, 256-262

American Diabetes Association. (2016). Standards of medical care in diabetes-2016. Diabetes Care, 39, S1.

Anderson, R. J., Freedland, K. E., Clouse, R. E. and Lustman, P. J. (2001). The prevalence of comorbid depression in adults with diabetes: a meta-analysis. Diabetes Care, 24, 1069-1078.

Bernal, H., Woolley, S., Schensul, J. J. and Dickinson, J. K. (2000). Correlates of self-efficacy in diabetes self-care among Hispanic adults with diabetes. The Diabetes Educator, 26, 673-680.

Bherer, L., Erickson, K. I. and Liu-Ambrose, T. (2013). A review of the effects of physical activity and exercise on cognitive and brain functions in older adults. Fournal of Aging Research, 2013, 1-8.

Black, S. A., Markides, K. S. and Ray, L. A. (2003). Depression predicts increased incidence of adverse health outcomes in older Mexican Americans with type 2 diabetes. Diabetes Care, 26, 2822-2828.

Cabassa, L. J. and Hansen, M. C. (2007). A systematic review of depression treatments in primary care for Latino adults. Research on Social Work Practice, 17, 494-503.

Center for Disease Control (2014). National Diabetes Statistics Report: Estimates of Diabetes and its Burden in the United States. Atlanta, GA: U.S. Department of Health and Human Services.

Cherrington, A., Wallston, K. A. and Rothman, R. L. (2010). Exploring the relationship between diabetes self-efficacy, depressive symptoms, and glycemic control among men and women with type 2 diabetes. Fournal of Behavioral Medicine, 33, 81-89.

Ciechanowski, P. (2011). Diapression: an integrated model for understanding the experience of individuals with co-occurring diabetes and depression. Clinical Diabetes, 29, 43-49.

Collado, A., Lim, A. C. and MacPherson, L. (2016). A systematic review of depression psychotherapies among Latinos. Clinical Psychology Review, 45, 193-209.

Cukierman-Yaffe, T. et al. (2009). Relationship between baseline glycemic control and cognitive function in individuals with type 2 diabetes and other cardiovascular risk factors the action to control cardiovascular risk in diabetes-memory in diabetes (ACCORD-MIND) trial. Diabetes Care, 32, 221-226. 
D'ath, P., Katona, P., Mullan, E., Evans, S. and Katona, C. (1994). Screening, detection and management of depression in elderly primary care attenders. I: the acceptability and performance of the 15 item Geriatric Depression Scale (GDS15) and the development of short versions. Family Practice, 11, 260-266.

Egede, L. E. and Osborn, C. Y. (2010). Role of motivation in the relationship between depression, self-care, and glycemic control in adults with type 2 diabetes. The Diabetes Educator, 36, 276-283.

Fontbonne, A., Berr, C., Ducimetière, P. and Alpérovitch, A. (2001). Changes in cognitive abilities over a 4-year period are unfavorably affected in elderly diabetic subjects: results of the epidemiology of vascular aging study. Diabetes Care, 24, 366-370.

Friedman, B., Heisel, M. J. and Delavan, R. L. (2005). Psychometric properties of the 15-item Geriatric Depression Scale in functionally impaired, cognitively intact, community-dwelling elderly primary care patients. Fournal of the American Geriatrics Society, 53, 1570-1576.

Gao, J. et al. (2013). Effects of self-care, self-efficacy, social support on glycemic control in adults with type 2 diabetes. BMC Family Practice, 14, 66-66.

Golden, S. H. (2007). A review of the evidence for a neuroendocrine link between stress, depression and diabetes mellitus. Current Diabetes Reviews, 3, 252-259.

Golden, S. H. et al. (2008). Examining a bidirectional association between depressive symptoms and diabetes. fAMA, 299, 2751-2759.

Hajat, A., Lucas, J. B. and Kington, R. (2000). Health outcomes among Hispanic subgroups: data from the National Health Interview Survey, 1992-95. Advance Data, $310,1-14$.

Harris, M. I., Eastman, R. C., Cowie, C. C., Flegal, K. M. and Eberhardt, M. S. (1999). Racial and ethnic differences in glycemic control of adults with type 2 diabetes. Diabetes Care, 22, 403-408.

Interian, A. and Díaz-Martínez, A. M. (2007). Considerations for culturally competent cognitive-behavioral therapy for depression with Hispanic patients. Cognitive and Behavioral Practice, 14, 84-97.

Kendzor, D. E. et al. (2014). The association of depression and anxiety with glycemic control among Mexican Americans with diabetes living near the US-Mexico border. BMC Public Health, 14, 176.

Kørner, A. et al. (2006). The geriatric depression scale and the Cornell scale for depression in dementia. A validity study. Nordic Fournal of Psychiatry, 60, 360-364.

Leroi, I., McDonald, K., Pantula, H. and Harbishettar, V. (2012). Cognitive impairment in Parkinson disease: impact on quality of life, disability, and caregiver burden. Fournal of Geriatric Psychiatry and Neurology, 25, 208-214. doi: 10.1177/08919887124648230891988712464823.

Lin, E. H. et al. (2010). Depression and advanced complications of diabetes a prospective cohort study. Diabetes Care, 33, 264-269.

Lincoln, A. K., Shepherd, A., Johnson, P. L. and Castaneda-Sceppa, C. (2011). The impact of resistance exercise training on the mental health of older Puerto Rican adults with type 2 diabetes. The fournals of Gerontology Series B: Psychological Sciences and Social Sciences, 66, 567-570.
Marioni, R. E. et al. (2010). Association between raised inflammatory markers and cognitive decline in elderly people with type 2 diabetes: the Edinburgh type 2 diabetes study. Diabetes, 59, 710-713.

Mceniry, M. and Palloni, A. (2010). Early life exposures and the occurrence and timing of heart disease among the older adult Puerto Rican population. Demography, 47, 23-43.

Meng, X. and D'Arcy, C. (2012). Education and dementia in the context of the cognitive reserve hypothesis: a systematic review with meta-analyses and qualitative analyses. PLoS One, 7, e38268.

Messier, C. (2005). Impact of impaired glucose tolerance and type 2 diabetes on cognitive aging. Neurobiology of Aging, 26, 26-30.

Mier, N. et al. (2008). Clinical depressive symptoms and diabetes in a binational border population. The fournal of the American Board of Family Medicine, 21, 223-233.

Munshi, M. et al. (2006). Cognitive dysfunction is associated with poor diabetes control in older adults. Diabetes Care, 29, 1794-1799.

Paterniti, S., Verdier-Taillefer, M.-H., DuFouil, C. and Alperovitch, A. (2002). Depressive symptoms and cognitive decline in elderly people longitudinal study. The British fournal of Psychiatry, 181, 406-410.

Pocklington, C., Gilbody, S., Manea, L. and McMillan, D. (2016). The diagnostic accuracy of brief versions of the geriatric depression scale: a systematic review and meta-analysis. International fournal of Geriatric Psychiatry, 31, 837-857.

Pottie, K. et al. (2015). Recommendations on screening for cognitive impairment in older adults. Canadian Medical Association fournal, 188, 37-46.

Richard, E. et al. (2013). Late-life depression, mild cognitive impairment, and dementia. $\mathcal{F} A M A$ Neurology, 70, 383-389.

Rivera-Hernandez, M., Leyva, B., Keohane, L. M. and Trivedi, A. N. (2016). Quality of care for White and Hispanic medicare advantage enrollees in the United States and Puerto Rico. $\mathcal{F A M A}$ Internal Medicine, 176, 787-794.

Roman, J. (2015). The Puerto Rico healthcare crisis. Annals of the American Thoracic Society, 12, 1760-1763.

Rosenberg, P. B., Mielke, M. M., Xue, Q.-L. and Carlson, M. C. (2010). Depressive symptoms predict incident cognitive impairment in cognitive healthy older women. The American fournal of Geriatric Psychiatry, 18, 204-211.

Rosselló, J., Bernal, G. and Rivera-Medina, C. (2008). Individual and group CBT and IPT for Puerto Rican adolescents with depressive symptoms. Cultural Diversity and Ethnic Minority Psychology, 14, 234-245.

Sacco, W. P., Wells, K. J., Vaughan, C. A., Friedman, A., Perez, S. and Matthew, R. (2005). Depression in adults with type 2 diabetes: the role of adherence, body mass index, and self-efficacy. Health Psychology, 24, 630-634.

Sánchez-Ayéndez, M. et al. (2003). Una escala psicométrica breve para evaluar el estado cognitivo de Hispanoparlantes de edad mayor. Puerto Rico Health Sciences fournal, 22, 377-384.

Sarkar, U., Fisher, L. and Schillinger, D. (2006). Is self-efficacy associated with diabetes self-management across race/ethnicity and health literacy? Diabetes Care, 29, 823-829. 
SAS Institute, Inc. (2013). SAS Software (Version 9.4). Cary, NC: SAS Institute, Inc.

Solanki, R., Dubey, V. and Munshi, D. (2009). Neurocognitive impairment and comorbid depression in patients of diabetes mellitus. International fournal of Diabetes in Developing Countries, 29, 133-138.

Steffens, D. C. et al. (2000). Prevalence of depression and its treatment in an elderly population: the Cache County study. Archives of General Psychiatry, 57, 601-607.

Stern, Y. (2013). Cognitive reserve: implications for assessment and intervention. Folia Phoniatrica et Logopaedica, 65, 49-54.

Strachan, M. W., Reynolds, R. M., Marioni, R. E. and Price, J. F. (2011). Cognitive function, dementia and type 2 diabetes mellitus in the elderly. Nature Reviews Endocrinology, 7, 108-114.

Takeda, S. et al. (2010). Diabetes-accelerated memory dysfunction via cerebrovascular inflammation and $\mathrm{A} \beta$ deposition in an Alzheimer mouse model with diabetes.
Proceedings of the National Academy of Sciences, 107, 7036-7041.

U.S. Census Bureau. (2011). The Older Population: 2010. Available at: https://www.census.gov/prod/cen2010/briefs/ c2010br-09.pdf; last accessed 28 May 2015.

Vera, M. et al. (2010). Collaborative care for depressed patients with chronic medical conditions: a randomized trial in Puerto Rico. Psychiatric Services, 61, 144-150.

Xu, W. et al. (2010). Accelerated progression from mild cognitive impairment to dementia in people with diabetes. Diabetes, 59, 2928-2935.

Yesavage, J. A. et al. (1983). Development and validation of a geriatric depression screening scale: a preliminary report. Fournal of Psychiatric Research, 17, 37-49.

Zahodne, L. B., Stern, Y. and Manly, J. J. (2014). Differing effects of education on cognitive decline in diverse elders with low versus high educational attainment. Neuropsychology, 29, 649-657. 